POSTER 17-26

\section{Public Perceptions of the Efficacy of Prehospital Cardiac Resuscitation}

\author{
*David R. Johnson, MD, FACEP, Joe Salgado, \\ $B S$, Robert Gougelet, MD \\ New Mexico Emergency Medical Services Academy, \\ Department of Emergency Medicine, University of \\ New Mexico, Albuquerque, New Mexico
}

Purpose: To determine if public expectations about the survival rate from prehospital cardiac arrest (PCA) is consistent with actual EMS system performance, and if knowledge of actual resuscitation rates influences an individual's desire to be resuscitated from PCA.

Methods: A questionnaire was administered to a convenience sample of individuals presenting to an urban teaching hospital emergency department. The questionnaire captured demographic data and an estimate of the probability of surviving a PCA in the local area. Individuals then were asked, before and after being informed of the actual local resuscitation rate, whether they would wish resuscitation from PCA. The actual resuscitation rate was calculated retrospectively from three years of ambulance run data and review of hospital records. Data were analyzed using the paired $t$-test and logistic regression.

Results: A total of 411 questionnaires were analyzed. Lay persons estimated the rate of successful PCA resuscitation to be $60.2 \pm 27.0 \%$ (mean \pm sd) compared to the actual rate of $6.5 \%$. When asked if they would wish resuscitation for themselves, $10.0 \%$ indicated "No" compared with $22.9 \%$ after being informed of the actual local resuscitation rate $(p=0.003)$. This correlated with age $(p=0.003)$ and prior expression of resuscitation wishes $(p=0.008)$.

Conclusions: The lay public greatly overestimates the success of resuscitation from PCA. After being informed of actual success rates, a significant portion of the public might prefer no resuscitation.
POSTER 18-28

\section{The Efficacy of Atropine in the Prehospital Treatment of Unstable Bradycardia and Atrioventricular Block}

Gary L. Swart, MD, *William J. Brady, Jr., MD, Daniel J. DeBehnke, MD

Medical College of Wisconsin, Milwaukee, Wisconsin

Objective: To evaluate the effectiveness of atropine in the prehospital treatment of unstable patients with bradycardia or atrioventricular block (AVB).

Methods: Design-Retrospective prehospital chart review, 1990-1992. Setting-Metropolitan fire-department-based paramedic system. Participants-One-hundred-seventy-two (172) adult patients receiving atropine for unstable bradycardia or AVB. Instability was defined according to advanced cardiac life support (ACLS) criteria. Data were grouped and analyzed according to both response to the last dose (RLD) of atropine received and initial rhythm (IR). RLD was grouped as: 1) adverse (ventricular arrhythmia); 2) none; 3) partial (systolic blood pressure [SBP] $<90 \mathrm{mmHg}$ or pulse $[\mathrm{P}]<60$ beats per minute, transient or maintained); or 4) complete ( $\mathrm{SBP}>90 \mathrm{mmHg}$ and $\mathrm{P}>60$ beats per minute, maintained). IR was classified as bradycardia (idioventricular, junctional, sinus) or AVB second degree [types I and II], third degree). Interventions-1987 ACLS protocol.

Results: Patients grouped according to RLD or IR were similar in demographics, medical history, degree of instability, field times, and treatment. RLD compared by IR was not significant. Complete responders more commonly received a single dose of atropine (Chi-square: $p=0.0002)$ and a lower total dose than did nonresponders $(0.7 \pm 0.4 \mathrm{mg}$ vs $1.1 \pm 0.6 \mathrm{mg}$ : paired two-tailed ttest; Tukey multiple comparisons method, $p<0.001$ ). Patients with a bradycardia more commonly received only a single dose of atropine (Chi-square: $p=0.03$ ), a lower total dose $(0.9 \pm 0.8$ vs. $1.1 \pm 0.6 \mathrm{mg}$, paired two-tailed $t$-test, $p=0.0006)$, and had an improved rhythm on hospital arrival compared to AVBs (Chi-square: $p=0.007$ ). Deaths occurred in the prehospital setting only in the adverse $(n=2)$ and no response $(n=14)$ groups.

Conclusions: In the prehospital setting, atropine is likely to be effective with the initial dose and is more likely to result in an improved rhythm when used in bradycardia than in AVB. 